\title{
Relationship between family meal frequency and individual dietary intake among diabetic patients
}

\author{
Divya Ruhee and Fawzi Mahomoodally*
}

\begin{abstract}
Background: Notoriously, the island of Mauritius has one of the highest prevalence of diabetes in the world. Management of the disease is very important and family meals are undoubtedly beneficial to patients as they promote the development of healthy eating behaviours and food choices. This study has aimed to probe into potential relationship(s) between family meal frequency and individual dietary intake among diabetic patients and to establish whether family cohesion may be a plausible mediator of this relationship.

Methods: A cross-sectional survey was carried out with a random sample of 384 diabetic patients. The Family Adaptability and Cohesion Evaluation Scale III was used to obtain information on two general aspects of family functioning, that is, cohesiveness and adaptability. Chi-squared $\left(X^{2}\right)$ tests, independent sample $t$-tests and one-way ANOVA were used to determine statistical significance. Pearson correlation was used to examine associations between family meal frequency, individual dietary intake and family cohesion. Hierarchical linear regression models were performed for the mediation analysis.

Results: Family meal frequency (breakfast, lunch and dinner) was observed to be positively associated with intake of fish, raw vegetables, dried and fresh fruits, low-fat milk, cheese, yogurt, nuts and light butter and negatively associated with intake of red meat, white rice, white bread, whole egg fried, chocolates, fried cakes, burgers, chips, and fried noodles/rice. Average mediation (52.6\%) was indicated by family cohesion for the association between family meal frequency and individual dietary intake among diabetic patients. Sobel's test further confirmed the trend towards complete mediation $(z=15.4 ; P<0.05)$.

Conclusions: A strong relationship between family meal frequency and individual dietary intake among diabetic patients was recorded. The present study is one of the few studies that have examined family cohesion as a mediator of the relationship and to our best knowledge is the first work to demonstrate a trend towards complete mediation. Results obtained can be used by health professionals to devise strategies for increasing knowledge and awareness of both diabetic patients and their respective families to curd down this public health burden.
\end{abstract}

Keywords: Diabetes, Individual dietary intake, Family meals, Family cohesion, Mediation analysis

\section{Background}

The World Health Organisation (WHO) defines diabetes as a chronic metabolic disease that occurs either when the pancreas does not produce enough insulin or when the body cannot effectively use the insulin it produces [33]. Diabetes is unfortunately one of the biggest public health challenges in human history and it is affecting even more people around the world each year. Even though the real

* Correspondence: f.mahomoodally@uom.ac.mu

Department of Health Sciences, Faculty of Science, University of Mauritius, 230 Réduit, Mauritius aetiology of diabetes is not fully understood by experts, the associated risk factors are numerous ranging from genetic triggers to environmental ones. It becomes imperative to detect and manage the disease as soon as possible because excessive fluctuations in blood glucose level can injure tissues throughout the whole body [31]. Most importantly there is strong evidence that the disease is the leading cause of cardiovascular diseases, renal problems, limb amputation, gangrene, peripheral nerve damage and visual impairment $[2,28]$. 
About 382 million people, in 2013, have diabetes in the world and it is expected to rise up to 592 million by 2035 if serious attention is not drawn towards the problem [14]. Mauritius is one of the countries with very high prevalence rate of diabetes and currently about 290,000 people suffer from diabetes with various health complications such as hypertension, renal failure and visual disorders [22]. Along with being a worrying situation in Mauritius, diabetes mellitus is equally associated with costs in terms of human suffering, health care and loss resources [22]. Hence, effective treatment for diabetes is of uttermost importance.

Pharmacotherapy is normally used by most diabetic patients as the primary means of achieving optimal glycaemic control. However, most drugs are usually associated with weight gain. Therefore, medical nutrition therapy (MNT) and lifestyle intervention remains the cornerstone for the management of diabetes, especially those with NIDDM [18]. Along with diet and exercise, the role of family functioning as a factor in the management of the disease has been suggested in many studies [26]. Various aspects of the family meal environment such as characteristics of the family during mealtimes, frequency of family meals and the atmosphere play a crucial part in modelling food-related behaviours [3].

Previous studies have focused mostly on the influence of family meals on the consumption of fruits and vegetables in adolescents. Moreover very few studies have been conducted on the relationship between family meal frequency and individual dietary intake and therefore there is no established theory to explain the association among diabetic patients in Mauritius. Family cohesion, defined as the emotional bonding that family members have towards one another, may have significant implications for the adoption of healthful eating habits [32]. It can therefore be hypothesised that the frequency of family meals reflects the family social environment and family cohesion, which may influence the individual dietary intake among diabetic patients. In the light of previous findings, it can also be hypothesised that greater frequency of family meals is associated with higher fruits and vegetables intake and lower consumption of high-calorie snacks, sweets and sweetened drinks among diabetic patients $[3,33]$. Henceforth, from all these studies and analyses, it can be said that there may be a relationship between family meals and family cohesion that had a direct impact on individual dietary intake. However, this relationship is not clear and need further investigation. Explaining the mechanism through which family meal frequency has a positive influence on individual dietary intake with cohesion as a possible mediator can help to intensify dietary interventions to improve the situation in Mauritius. The purpose of this project is therefore to determine whether there is a relationship between family meal frequency and individual dietary intake among diabetic patients in Mauritius and to examine family cohesion as a possible mediator of this relationship.

\section{Methods}

Design

A self-administered cross-sectional survey was conducted to meet the objectives of the study since it is a quantitative data collection approach targeted to obtain information from a representative sample of the population. For a population of about 290,000 diabetic patients in Mauritius, the requested sample size, for \pm precision with $95 \%$ confidence interval $(\mathrm{CI})$ and degree of variability, p equal to 0.5 , was found to be 384 . To make the sample representative of the population suffering from diabetes mellitus, participants were recruited randomly from health care centres all around the island from both rural and urban areas. Nongovernmental organizations such as Mauritius Diabetes Association, T1diams, Diabetes Parent Support Group and 'Groupement des Diabétiques' were also targeted.

The respondents selected for the study had to meet the following inclusion criteria: (1) Type I and Type II diabetic patients only were recruited; (2) patients above 12 years; (3) Participants who are mentally fit to answer questions; and (4) Participants should be of Mauritian origin and they should be able to understand English, French and Creole (vernacular) language. The exclusion criteria included patients suffering from other types of diabetes, except Type I and Type II. Diabetic patients suffering from extremely poor health conditions and having difficulties to understand and answer questions were also excluded.

\section{Design and content of questionnaire}

The questionnaire comprised of 20 main questions and divided into four distinct sections; personal data such as socio-demographic information; individual dietary intake; family meal frequency and family support and cohesion [13].

Section A consisted of questions based on personal and socio-demographic information such as age, sex, ethnic group, residential area, educational background, occupation, income group, marital status and number of persons in the family which are considered to be predictors of self care behaviour. Questions on types of diabetes, family history of diabetes and body mass index (BMI) were also being asked. BMI was calculated as weight $(\mathrm{kg}) /$ height squared $\left(\mathrm{m}^{2}\right)$. The weight of participants, with their shoes removed, was recorded by the first author using a calibrated digital scale and the height was measured using a measuring tape.

Section B consisted of questions based on individual dietary intake such as vegetarianism, number of meals taken each day, skipping meals and glycaemic index. Dietary intake of diabetic patients was assessed using a self-administered food frequency questionnaire adapted from the Diet History Questionnaire [32]. In this study, 
the food frequency questionnaire was modified and adapted. For instance, the questionnaire was translated to French and to the local vernacular language 'creole'. Prior to start of the full-scale survey, a pilot testing was conducted with ten subjects, in order to judge the clarity of the questions. A few amendments were then brought to the questionnaire, where some of the questions were rephrased due to ambiguity. Response options consisted of 5 categories such as never, 1-2 times, 3-4 times, 5-6 times and everyday and they were recorded to reflect an average frequency of consumption per week such as 0 , 1.5, 3.5, 5.5 and 7 times per week. In addition, participants were also asked to report their average serving size of consumed items which consisted of four categories of response options such as $1 / 2$ cup, 1 cup, 2 cups and more than 2 cups and average sizes were recorded as $0.5,1,1.5$ and 2 servings respectively. The average number of servings per week of given items was calculated by multiplying frequency of consumption by average servings [32].

Section $C$ was based on family meal frequency which was assessed by the question, "In the past week, how many times did you and your family (living in your house) had breakfast, lunch and dinner together?" Responses were accepted as never, 1-2 times, 3-4 times and every day.

Section D consisted of questions on family support and cohesion. The Family Adaptability and Cohesion Evaluation Scale III (FACES III) is a validated 20-item self-reporting scale which was used to obtain information on two general aspects of family functioning, that is, cohesiveness and adaptability [12 http://www.eruralfamilies.org]. Family cohesion assesses degree of separation or connection of family members to the family. There are four levels of family cohesion ranging from extreme low cohesion to extreme high cohesion- disengaged, separated, connected, and enmeshed. There are four levels of adaptability - rigid, structured, flexible, and chaotic [21]. Through various studies conducted, it became well known that cohesiveness is a more predictive and powerful variable than adaptability [21]. Therefore only the cohesion dimension was used in the analysis of the study. The cohesion scale of FACES-III (see Table 1) contained 10 items. Participants were asked to describe their family or household as it was by checking the appropriate number in the boxes for each item. All the responses were recorded on a five-point scale ranging from 1 ("almost

Table 1 Characteristics and interpretation of the Family Adaptability and Cohesion Evaluation Scale III

\begin{tabular}{lll}
\hline Subscale & Scoring range & Interpretation \\
\hline Cohesion scale & $10-50$ & $10-34:$ Disengaged family \\
& $35-40:$ Separated family \\
& $41-45:$ Connected family \\
& $46-50:$ Enmeshed family \\
\hline
\end{tabular}

never") to 5 ("almost always"). Therefore the lowest possible score is 10 and the maximum score is 50 , and the higher the score, the greater perceived family cohesion [21].

\section{Data collection}

The questionnaire was designed in English language and translated in Creole, a commonly spoken local language in Mauritius, or French for those who were not comfortable with English language. However, in cases where participants had language and communication issues, data was collected using face to face interviews where the questionnaire was filled out by the first author. Participation to the study was voluntary and formal consent was obtained from all the respondents. An informed consent form was provided at the beginning of each questionnaire where they were assured of the confidentiality of the data collected [7, 17].

\section{Statistical analysis of data}

Data was analysed using SPSS (version 16.0) software and Excel 2007. Chi squared $\left(\chi^{2}\right)$ test, independent sample $\mathrm{t}$-tests and one-way ANOVA analysis were performed to compare the various socio-demographic variables, family cohesion scores and eating behaviours of diabetic patients. Pearson correlation coefficients were used to examine the associations between family cohesion and family meal frequency. The Baron and Kenny approach as described by Welsh et al. [32] was the main statistical method used in this study. There were four steps that were adopted to prove the presence of a mediator, that is, family cohesion:

1. To demonstrate that the independent variable $X$ (family meal frequency) was associated with the outcome variable Y (individual dietary intake).

2. To demonstrate that the independent variable $X$ was associated with the mediator variable $M$ (family cohesion).

3. To demonstrate that the mediator variable $M$ was associated with the outcome variable $\mathrm{Y}$ after controlling for X.

4. The last step consisted of determining whether the effect of $\mathrm{X}$ on $\mathrm{Y}$ was reduced when $\mathrm{M}$ was included in a regression model, providing evidence that the effect of $\mathrm{X}$ on $\mathrm{Y}$ was partially mediated by $\mathrm{M}$. Furthermore, if the path between $\mathrm{X}$ and $\mathrm{Y}$ disappeared completely when $\mathrm{M}$ was included, complete mediation by $\mathrm{M}$ was indicated [see Fig. 1].

A series of hierarchical multiple linear regression models were performed where each diabetic patient's average weekly servings of a targeted food or beverage item were regressed on frequency of family meals and included a random effect for household (family cohesion). 


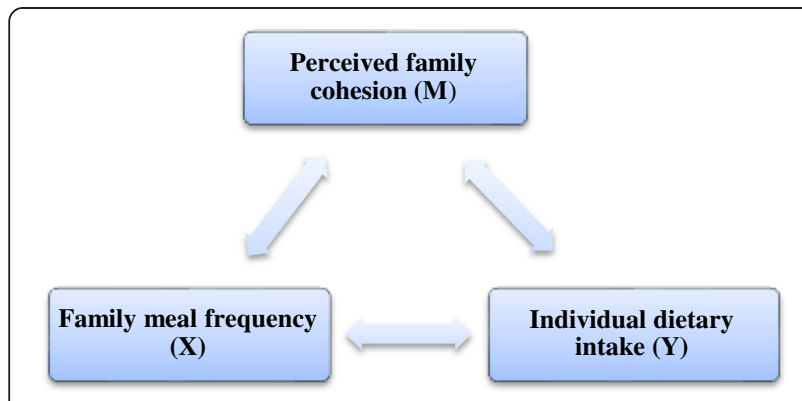

Fig. 1 Associations between the different variables in the study

Similar regression models were fit regressing family cohesion scores on frequency of family meals as well as regressing average servings of targeted food or beverage items on family cohesion scores after controlling for frequency of family meals. Regression models regressing weekly servings of a targeted food category on both family meal frequency and family cohesion score were fit in order to provide evidence for mediation. When mediation was indicated, the percentage reduction for the association of family meals and individual dietary intake as mediated by family cohesion was calculated by subtracting the adjusted regression coefficient for family meal frequency from its unadjusted counterpart and then dividing by the unadjusted regression coefficient for frequency of family meals [32]. For each of those tests, a $P$ value of less than 0.05 was considered as statistically significant.

\section{Results}

\section{Demographic profile}

A total of 384 participants completed the questionnaire which was representative of the Mauritian population. Socio-demographic variables such as gender, age group, ethnic group, demographic area, marital status, educational background, occupation, income group, number of persons in family, type of diabetes and family history of diabetes are displayed in Table 2.

\section{Anthropometric measurement}

The BMI was calculated from the measured height and weight of the respondents and Fig. 2 illustrates the number of individuals, in the different BMI categories. As it can be seen $53.6 \%$ of the respondents were normal with a BMI of "18.5-24.9" whereas the rest was either underweight or overweight or obese carrying a percentage of $12.0 \%, 29.4 \%$ and $4.9 \%$ respectively which tend to corroborate with actual figures depicted by the growing number of overweight and obese people in Mauritius.

\section{Individual dietary intake}

The eating behaviours and food consumption of the participants were assessed using different questions on vegetarianism, number of meals per day, skipping meals and knowledge of glycaemic index. The Food Frequency Questionnaire indicated the food intake of specific food items. It was found that $99.0 \%$ of the subjects were non-vegetarian and only $1.0 \%$ were vegetarian. $52.6 \%$ consumed " 3 meals" per day while the rest had "less than 3 meals", "5-7 meals" and "more than 7 meals" with percentage occurrence of 9.4\%, $28.9 \%$ and $9.1 \%$ respectively (see Table 3). $60.7 \%$ of the respondents do not skip meals whereas the remaining $39.3 \%$ do skip meals.

It was observed that $97.4 \%$ of the participants were ignorant about the meaning of "glycaemic index" and only $2.6 \%$ were aware of it. Participant-reported food consumption of the different food items are shown in Table 4. Statistical comparisons of the mean servings per week of the food items demonstrated that the respondents had a higher consumption of rice (white), bread (white), raw vegetables, fresh fruits and water with mean values of $6.24,6.33,6.06,6.26$ and 7.25 respectively. Lower consumption was recorded for beef, pork, liver/organ meat, dholl-puri/farata, whole egg boiled, egg without yolk, root vegetables, dried fruits, chocolates, pastries, gateaux-piments, light butter, diet drinks and artificial sweeteners with mean values of $0.191,0.110,1.32,1.63,1.55,1.13,1.57,1.82,1.63$, $0.93,1.81,1.99,0.457$ and 0.74 respectively.

\section{Family meal frequency}

The frequency of family meals was reported by participants in three categories namely "eat breakfast together", "eat lunch together" and "eat dinner together" and summarised in Table 5. The percentage of respondents who never ate breakfast together as a family was $50.8 \%$ and $56.2 \%$ never ate lunch as a family.

\section{Family cohesion}

Family cohesion was measured using the cohesion scale from the Family Adaptability and Cohesion Evaluation Scale III (FACES-III). The majority of the respondents were from a disengaged family (i.e. extreme low cohesion) with an occurrence of $51.6 \%$. $26.6 \%$ came from an enmeshed family, $14.1 \%$ from a connected family and $7.8 \%$ from a separated family (see Fig. 3). The mean value and standard deviation of the family cohesion score (FACES-III cohesion score) was $31.9 \pm 12.7$ showing an average perceived family cohesion.

\section{Socio-demographic variables and BMI}

A strong statistically significant relationship $(P<0.05)$ between BMI and the following socio-demographic variables (age group, ethnic group, demographic area, educational background, occupation and family history) was recorded. However, no significance was 
Table 2 Descriptive statistics for socio-demographic variables

\begin{tabular}{|c|c|c|}
\hline Socio-demographic Variables & $\mathrm{n}$ & $\%$ \\
\hline \multicolumn{3}{|l|}{ Gender } \\
\hline Male & 208 & 54. \\
\hline Female & 176 & 45 \\
\hline \multicolumn{3}{|l|}{ Age group } \\
\hline $12-17$ & 44 & 11. \\
\hline $18-30$ & 59 & 15 \\
\hline $30-60$ & 208 & 54 \\
\hline$>60$ & 73 & 19 \\
\hline \multicolumn{3}{|l|}{ Ethnic group } \\
\hline Christian & 102 & 26. \\
\hline Muslim & 65 & 16 \\
\hline Hindu & 187 & 48 \\
\hline Chinese & 28 & 7.3 \\
\hline General Population & 2 & 0.5 \\
\hline \multicolumn{3}{|l|}{ Demographic area } \\
\hline Urban & 238 & 62. \\
\hline Rural & 146 & 38 \\
\hline \multicolumn{3}{|l|}{ Marital status } \\
\hline Married & 234 & 60. \\
\hline Single & 150 & 39. \\
\hline \multicolumn{3}{|l|}{ Educational background } \\
\hline None & 2 & 0.5 \\
\hline Primary & 71 & 18 \\
\hline Secondary & 190 & 49 \\
\hline Tertiary & 121 & 31. \\
\hline \multicolumn{3}{|l|}{ Occupation } \\
\hline Student & 58 & 15. \\
\hline Manual Worker & 42 & 10 \\
\hline Housewife/Unemployed & 82 & 21. \\
\hline Office/managerial & 147 & 38 \\
\hline Others & 63 & 13. \\
\hline \multicolumn{3}{|l|}{ Income group } \\
\hline$<$ Rs. 10,000 & 145 & 37. \\
\hline Rs.10,000-Rs. 20,000 & 161 & 41. \\
\hline$>$ Rs. 20,000 & 78 & 20. \\
\hline \multicolumn{3}{|l|}{ Number of persons in family } \\
\hline 1 & 8 & 2.1 \\
\hline $2-3$ & 154 & 40. \\
\hline $4-6$ & 168 & 43. \\
\hline More than 6 & 54 & 14. \\
\hline \multicolumn{3}{|l|}{ Type of Diabetes } \\
\hline Type 1 & 82 & 21. \\
\hline Type 2 & 302 & 78. \\
\hline
\end{tabular}

Table 2 Descriptive statistics for socio-demographic variables (Continued)

\begin{tabular}{lll}
\hline Yes & 290 & 75.5 \\
No & 94 & 24.5 \\
Member of family with diabetes & & \\
Mother & 82 & 21.4 \\
Father & 94 & 24.5 \\
Mother and Father & 65 & 16.9 \\
Grandparents & 49 & 12.8 \\
N/A & 94 & 24.5 \\
\hline
\end{tabular}

recorded between BMI with respect to the following demographic variables; sex $(P=0.554)$, marital status $(P=0.913)$, income group $(P=0.119)$, number of persons in family $(P=0.104)$ and type of diabetes $(P=0.973)$.

Socio-demographic variables and number of meals per day A strong significant relationship between age group $(P=0.000)$, occupation $(P=0.000)$, income group $(P=0.000)$ and type of diabetes $(P=0.001)$ with respect to number of meals per day was recorded. However no statistically significant relationship was noted for sex $(P=0.367)$, ethnic group $(P=0.054)$, demographic area $(P=0.060)$, marital status $(P=0.099)$, educational background $(P=0.126)$, number of persons in family $(P=0.347)$ and family history $(P=0.562)$ with respect to number of meals per day.

\section{Socio-demographic variables and skipping meals}

A strong statistically significant relationship between age group $(P=0.000)$ and educational background $(P=0.000)$ with respect to skipping meals was recorded. However, no statistically significant relationship was noted for sex $(P=0.612)$, ethnic group $(P=0.078)$, demographic area $(P=0.341)$, marital status $(P=0.893)$, occupation $(P=0.073)$, income group $(P=0.674)$, type of diabetes $(P=0.075)$ and family history $(P=0.088)$ with respect to skipping meals.

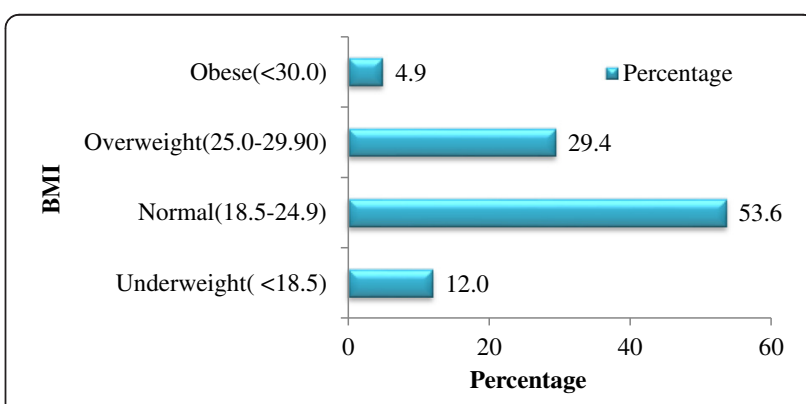

Fig. 2 Percentage of the different categories of BMI 
Table 3 Descriptive statistics of eating behaviours of subjects

\begin{tabular}{lll}
\hline Eating behaviour & $\mathrm{n}$ & $\%$ \\
\hline Vegetarianism & 4 & 1.0 \\
Yes & 380 & 99.0 \\
No & & \\
Number of meals/day & 202 & 52.6 \\
$<3$ & 36 & 9.4 \\
3 & 111 & 28.9 \\
$5-7$ & 35 & 9.1 \\
More than 7 & & \\
Skipping meals & 151 & 39.3 \\
Yes & 233 & 60.7 \\
No & & \\
Glycaemic index & 10 & 2.6 \\
Yes & 374 & 97.4 \\
No & &
\end{tabular}

\section{Socio-demographic variables and glycaemic index}

A strong statistically significant relationship between age group $(P=0.001)$, occupation $(P=0.000)$, type of diabetes $(P=0.001)$ and family history $(P=0.008)$ with respect to glycaemic index was recorded. However no statistically significant relationship was found between sex $(P=0.454)$, ethnic group $(P=0.093)$, demographic area $(P=0.212)$, marital status $(P=0.562)$, educational background $(P=0.229)$, income group $(P=0.208)$, number of persons in family $(P=0.520)$ with respect to glycaemic index.

\section{Socio-demographic variables and average number of servings per week}

A significantly strong relationship between ethnic group and chicken consumption $(P=0.001)$, beef $(P=0.000)$ and fish $(P=0.001)$ was recorded. Moreover, statistically strong relationship was found between demographic area and chicken $(P=0.000)$ and lamb $(P=0.000)$ consumption. Strong relationships were also found between income group and lamb $(P=0.001)$, brown bread $(P=0.001)$ and burger consumption $(P=0.001)$. Interestingly, the results also revealed strong statistically significant relationship between age group and burger $(P=0.000)$, chips $(P=0.000)$, pizza $(P=0.020)$, briani $(P=0.009)$ and fried noodles/rice consumption $(P=0.046)$. Strong relationships were equally found between educational background and burger $(P=0.000)$, chips $(P=0.000)$ and pizza consumption $(P=0.000)$. However there was no statistically significant relationship between the socio-demographic variables and the other remaining food items.

\section{Association between family meal frequency and family cohesion score}

Table 6 shows the association between family meal frequency and family cohesion score. A very strong positive correlation between "eat breakfast together" $(r=0.850)$, "eat lunch together" ( $r=0.895)$ and "eat dinner together" $(r=0.827)$ with respect to family cohesion score was recorded.

\section{Association between average number of servings per week of food items (individual dietary intake) and family cohesion score}

Strong negative correlation between lamb/mutton $(r=-0.631)$, white rice $(r=-0.610)$, whole egg fried $(r=-0.644)$, gateaux-piments $(r=-0.674)$, burger $(r=-0.657)$, chips $(r=-0.669)$, briani $(r=-0.671)$ and fried noodles/rice $(r=-0.677)$ consumption with respect to perceived family cohesion score was recorded. Moreover, strong positive association was found between egg without yolk $(r=0.659)$, dried fruits $(r=0.731)$, low-fat milk $(r=0.682)$, cheese $(r=0.611)$, yogurt $(r=0.671)$, light butter $(r=0.627)$ with respect to perceived family cohesion score. Interestingly, very strong correlations were found between fish $(r=0.839)$, raw vegetables $(r=0.804)$, fresh fruits $(r=0.807)$ and nuts $(r=0.877)$ consumption with respect to family cohesion scores.

\section{Association between family meal frequency and individual dietary intake}

For those who had breakfast together, there was a strong negative association ranging from -0.643 to -0.716 with respect to family meal frequency. It was also noted that there was a strong positive correlation between raw vegetables ranging from 0656 to 0.788 with respect to family meal frequency. In addition, results equally demonstrated a very strong positive association between fish $(r=0.863)$ and nuts $(r=0.893)$ consumption with respect to family meal frequency. For those who ate lunch together, there was a strong negative association between lamb/mutton $(r=-0.622)$, white rice $(r=-0.658)$, white bread $(r=-0.681)$, whole egg fried $(r=-0.633)$, gateauxpiments $(r=-0.691)$, burger $(r=-0.666)$, chips $(r=-0.676)$, briani $(r=-0.681)$ and fried noodles/rice $(r=-0.694)$ consumption with respect to family meal frequency. There was a statistically strong positive correlation between egg without yolk $(r=0.721)$, dried fruits $(r=0.709)$, low-fat milk $(r=0.652)$, yogurt $(r=0.681)$ and light butter $(r=0.658)$ consumption with respect to family meal frequency. It was also seen that there was a very strong positive association between fish $(r=0.873)$, raw vegetables $(r=0.875)$, fresh fruits $(r=0.863)$ and nuts $(r=0.919)$ consumption with respect to family meal frequency. For those diabetic participants who 
Table 4 Average number of servings per week of the given food items from food frequency questionnaire

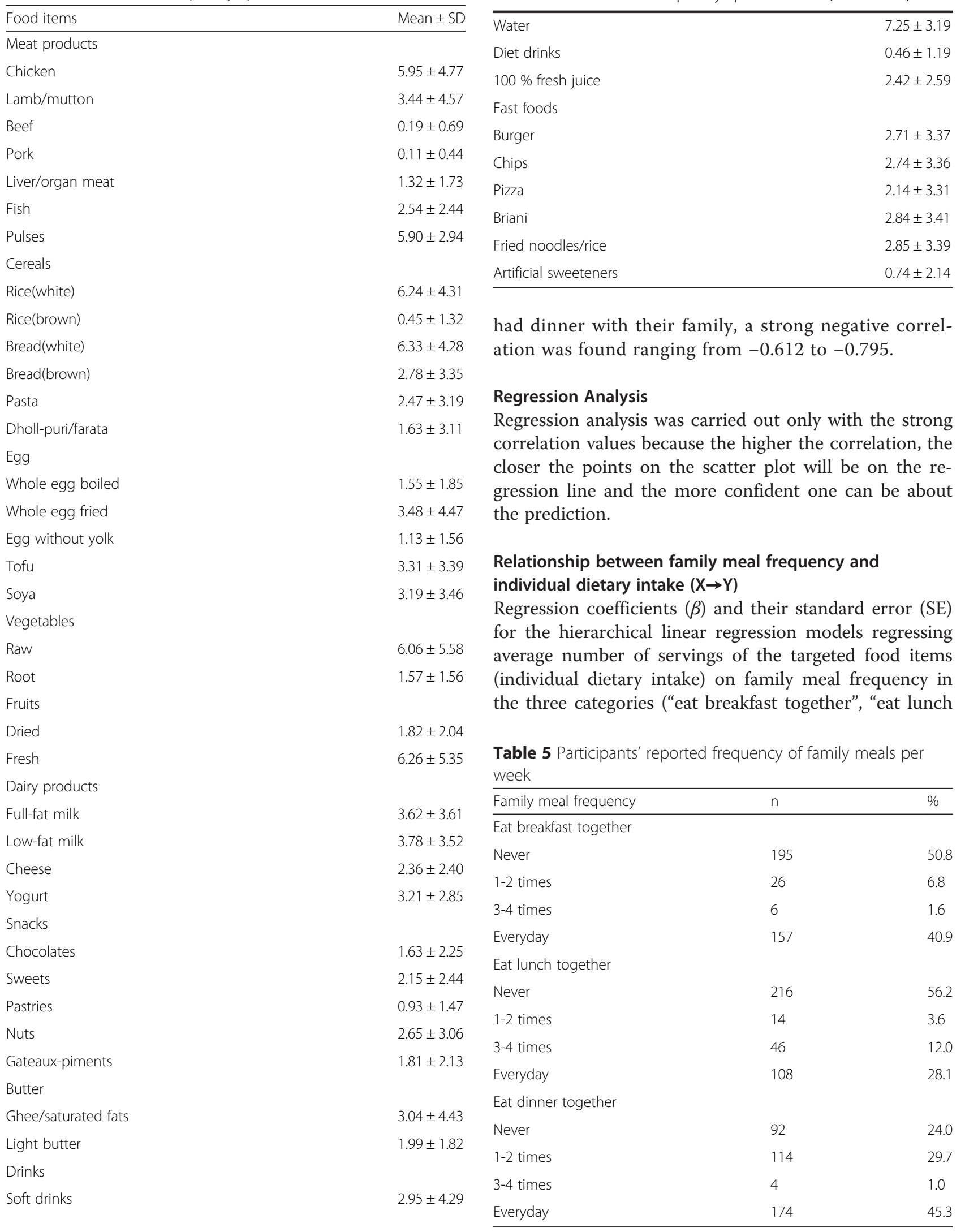

Table 4 Average number of servings per week of the given food items from food frequency questionnaire (Continued) 


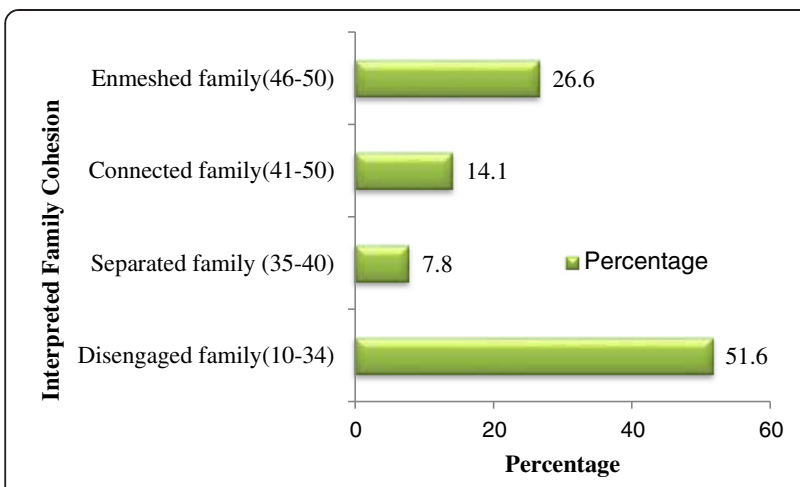

Fig. 3 Percentage of the interpreted family cohesion from FACES-III

together" and "eat dinner together") are displayed in Table 7.

\section{Relationship between family meal frequency and family cohesion $(\mathrm{X} \rightarrow \mathrm{M})$}

Regression coefficients $(\beta)$ and their standard error (SE) for the hierarchical linear regression models regressing family cohesion scores on family meal frequency in the three categories ("eat breakfast together", "eat lunch together" and "eat dinner together") was carried out. The family cohesion score demonstrated strong positive associations when regressed on family meal frequency $(p<0.005)$.

\section{Relationship between family cohesion and individual dietary intake $(\mathrm{M} \rightarrow \mathrm{Y})$}

Regression coefficients $(\beta)$ and standard error (SE) for hierarchical linear regression models regressing average number of servings of the targeted food items on family cohesion scores was carried out. Significant positive associations for family cohesion scores and fish $(\beta=0.161)$, egg without yolk $(\beta=0.081)$, raw vegetables $(\beta=0.352)$, dried fruits $(\beta=0.117)$, fresh fruits $(\beta=0.340)$, low-fat milk $(\beta=0.189)$, cheese $(\beta=0.115)$, yogurt $(\beta=0.150)$, nuts $(\beta=0.211)$ and light butter $(\beta=0.090)$ were observed.

Significant negative associations were found for family cohesion scores and lamb/mutton $(\beta=-0.227)$, white rice $(\beta=-0.207)$, whole egg fried $(\beta=-0.226)$, burger $(\beta=-0.174)$, chips $(\beta=-0.176)$, briani $(\beta=-0.180)$ and fried noodles/rice $(\beta=-0.180)$.

Table 6 Association between family meal frequency and family cohesion score

\begin{tabular}{lc}
\hline Family meal frequency & Family cohesion score $(r)$ \\
\hline Eat breakfast together & $0.850^{* *}$ \\
Eat lunch together & $0.895^{* *}$ \\
Eat dinner together & $0.827^{* *}$ \\
\hline
\end{tabular}

**Correlation is significant at the 0.01 level

\section{Mediation Analysis $(\mathrm{X} \rightarrow \mathrm{M} \rightarrow \mathrm{Y})$}

Table 8 shows the regression coefficients $(\beta)$ and standard error (SE) for hierarchical linear regression models regressing average number of servings of the targeted food items on family cohesion scores, after controlling for frequency of family meals. It can be deduced that significant positive associations continued to be observed for family cohesion scores and intake of fish, egg without yolk, raw vegetables, dried fruits, fresh fruits, low-fat milk, cheese, yogurt and nuts after controlling for frequency of family meals, that is, "eat breakfast together", "eat lunch together" and "eat dinner together".

These findings indicate a $52.6 \%$ reduction for the association of family meal frequency and individual dietary intake of the targeted food items as mediated by family cohesion. The calculation was done by subtracting the adjusted regression coefficient for family meal frequency from its unadjusted counterpart and then dividing by the unadjusted regression coefficient for frequency of family meals. The percentage reduction obtained clearly indicated a more or less complete mediation (>50\%) for the association between family meal frequency and individual dietary intake as mediated by family cohesion. Sobel's test, carried out using SPSS version 16.0, confirmed the trend towards complete mediation by family cohesion for the relationship between family meal frequency and individual dietary intake as indicated with $\mathrm{z}=15.4$ and $P=0.000$.

\section{Discussion}

The main aim of this study was to examine the relationship between family meal frequency and individual dietary intake among diabetic patients in Mauritius. In addition to the primary aim, it was also hypothesised that family cohesion may be a plausible mediator of this relationship. To our best of knowledge, no such studies have evaluated the association between family meal frequency, individual dietary intake and family cohesion among diabetic patients in Mauritius. It was hypothesised that family meal frequency would be positively associated with healthy food items intake (chicken, fish, liver/organ meat, pulses, brown rice, brown bread, boiled egg, egg without yolk, tofu, soya, vegetables, fruits, nuts, low-fat milk, cheese, yogurt, light butter, fresh juice, water and artificial sweeteners) and negatively associated with less healthy food items intake (lamb/mutton, beef, white rice, white bread, dholl-puri/farata, full-fat milk, oily and sugary snacks, ghee/saturated fats, soft drinks and fast foods). Moreover, an endeavour has been made to assess a series of socio-demographic variables including sex, age group, ethnic group, demographic area, marital status, educational background, occupation, income group, number of family members, type of diabetes and family history of diabetes in relation to 
Table 7 Regression coefficients for average food servings per week regressed on family meal frequency

\begin{tabular}{|c|c|c|c|c|c|c|}
\hline \multirow{3}{*}{$\begin{array}{l}\text { Dependent Variable(Y) } \\
\text { Average servings/week }\end{array}$} & \multicolumn{6}{|c|}{ Independent Variable $(X)$} \\
\hline & \multicolumn{2}{|c|}{ Eat breakfast together } & \multicolumn{2}{|c|}{ Eat lunch together } & \multicolumn{2}{|c|}{ Eat dinner together } \\
\hline & $\overline{\beta^{a} \pm S E^{b}}$ & $y$-intercept & $\overline{\beta^{a} \pm S E^{b}}$ & $y$-intercept & $\overline{\beta^{\mathrm{a}} \pm S E^{\mathrm{b}}}$ & $y$-intercept \\
\hline Lamb/mutton & $-2.16 \pm 0.120^{*}$ & 8.47 & $-2.12 \pm 0.137^{*}$ & 7.93 & $-2.19 \pm 0.147^{*}$ & 9.29 \\
\hline Fish & $1.47 \pm 0.044^{*}$ & -0.88 & $1.59 \pm 0.046^{*}$ & -0.83 & $1.44 \pm 0.066^{*}$ & -1.29 \\
\hline Rice(white) & $-1.86 \pm 0.121^{*}$ & 10.6 & $-2.12 \pm 0.124^{*}$ & 10.7 & $-2.08 \pm 0.138^{*}$ & 11.8 \\
\hline Bread(white) & $-1.99 \pm 0.114^{*}$ & 10.9 & $-2.17 \pm 0.120^{*}$ & 10.9 & - & - \\
\hline Whole egg fried & $-2.01 \pm 0.122^{*}$ & 8.14 & $-2.11 \pm 0.132^{*}$ & 7.95 & - & - \\
\hline Egg without yolk & $3.06 \pm 0.122^{*}$ & -1.06 & $0.84 \pm 0.041^{*}$ & -0.64 & - & - \\
\hline Raw vegetables & $1.11 \pm 0.045^{*}$ & -0.77 & $3.64 \pm 0.103^{*}$ & -1.65 & $3.04 \pm 0.163^{*}$ & -2.07 \\
\hline Dried fruits & $2.91 \pm 0.120^{*}$ & -0.51 & $1.08 \pm 0.055^{*}$ & -0.47 & $1.04 \pm 0.062^{*}$ & -0.98 \\
\hline Fresh fruits & $1.61 \pm 0.095^{*}$ & 0.03 & $3.45 \pm 0.103^{*}$ & -1.04 & $2.93 \pm 0.156^{*}$ & -1.58 \\
\hline Low-fat milk & $1.25 \pm 0.057^{*}$ & -0.53 & $1.71 \pm 0.102^{*}$ & 0.14 & - & - \\
\hline Cheese & $1.39 \pm 0.073^{*}$ & -0.02 & - & - & $1.16 \pm 0.076^{*}$ & -0.75 \\
\hline Yogurt & $-1.01 \pm 0.061^{*}$ & 3.98 & $1.45 \pm 0.080^{*}$ & 0.14 & - & - \\
\hline Nuts & $1.91 \pm 0.049^{*}$ & -1.79 & $2.09 \pm 0.046^{*}$ & -1.80 & $1.93 \pm 0.074^{*}$ & -2.52 \\
\hline Gateaux-piments & $-1.06 \pm 0.053^{*}$ & 4.29 & $-1.09 \pm 0.059^{*}$ & 4.14 & $-1.23 \pm 0.059^{*}$ & 5.09 \\
\hline Light butter & $0.96 \pm 0.043^{*}$ & -0.23 & $0.89 \pm 0.052^{*}$ & 0.09 & $0.90 \pm 0.057^{*}$ & -0.42 \\
\hline Burgers & $-1.68 \pm 0.084^{*}$ & 6.61 & $-1.67 \pm 0.096^{*}$ & 6.25 & $-2.06 \pm 0.086^{*}$ & 8.22 \\
\hline Chips & $-1.71 \pm 0.082^{*}$ & 6.73 & $-1.69 \pm 0.094^{*}$ & 6.33 & $-2.07 \pm 0.084^{*}$ & 8.28 \\
\hline Briani & $-1.59 \pm 0.090^{*}$ & 6.56 & $-1.73 \pm 0.095^{*}$ & 6.51 & $-2.14 \pm 0.084^{*}$ & 8.56 \\
\hline Fried noodles/rice & $-1.63 \pm 0.080^{*}$ & 6.64 & $-1.76 \pm 0.093^{*}$ & 6.57 & $-2.16 \pm 0.081^{*}$ & 8.63 \\
\hline
\end{tabular}

Note: all regression coefficients were adjusted for participant age and sex * Significance is indicated with $P$-value $<0.05$

${ }^{\mathrm{a}}$ Regression coefficient. ${ }^{\mathrm{b}}$ Standard error

glycaemic index, skipping meals, number of meals per day, intake of targeted food items and BMI.

This study has shown that $40.9 \%, 28.1 \%$ and $45.3 \%$ of the diabetic patients had breakfast together, eat lunch and dinner together respectively. Mellin et al. [20] have found that $57 \%$ of families have regular and frequent family meals which were more or less in line with our findings. Additionally, results have also suggested that greater frequency of family meals was significantly associated with greater average servings of fish, raw vegetables, dried fruits, fresh fruits, low-fat milk, cheese, yogurt, nuts and light butter. Greater family meal frequency demonstrated a trend towards lower average servings of lamb/mutton, white rice, white bread, whole egg fried, chocolates, gateaux-piments, burgers, chips, briani and fried noodles/rice. Previous studies by Andaya et al. [3] have shown that children consuming breakfast, lunch and dinner with their family at least 4 days per week had a percentage intake of $84 \%, 85 \%$ and $80 \%$ of fruits and vegetables respectively. Moreover, the study supported our findings by indicating only a $40 \%$, $44 \%$ and $43 \%$ of soda and chips consumption when children ate breakfast, lunch and dinner with their families respectively. Positive associations between regular family meals and daily intake of vegetables, calcium-rich food, fibre, calcium, magnesium, potassium, zinc, folate, vitamin $A$ and $B_{6}$ and negative associations with fast- food intake were also found by Burgess-Champoux et al. [5]. Another study by Larson et al. [17], which was in line with our results, reported that eating dinner was significantly associated with higher dietary intakes of vegetables and fruits. They equally reported that young adults enjoyed and valued eating with other family members. Fulkerson et al. [10] have demonstrated that family dinner frequency was positively associated with fruit consumption. Therefore our present findings were somewhat consistent with previous literature.

To our best of knowledge, no such studies have examined the association between family cohesion, family meal frequency and dietary intake among diabetic patients. Studies previously done on family cohesion are relatively few and none of them have really investigated on dietary consumption. Combrey et al. [10] have shown that adolescent reported overeating was associated with lower scores of family cohesion and adaptability. Significant negative associations were indicated by Patton et al. [26] between the dietary adherence of children and two dimensions of family function (task accomplishment and behavioural control). 
Table 8 Regression coefficients regressing average number of number of servings (individual dietary intake) on family cohesion scores and family meal frequency

\begin{tabular}{|c|c|c|c|c|c|c|}
\hline \multirow{3}{*}{$\begin{array}{l}\text { Dependent Variable(Y)- Average } \\
\text { number of servings/week }\end{array}$} & \multicolumn{6}{|c|}{ Independent Variable } \\
\hline & \multicolumn{2}{|c|}{ Eat breakfast together } & \multicolumn{2}{|c|}{ Eat lunch together } & \multicolumn{2}{|c|}{ Eat dinner together } \\
\hline & $\beta^{\mathrm{a}} \pm S E^{\mathrm{b}}$ & $\overline{y \text {-intercept }}$ & $\beta^{a} \pm S E^{b}$ & $y$-intercept & $\beta^{a} \pm S E^{b}$ & $y$-intercept \\
\hline \multirow[t]{2}{*}{ Lamb/mutton } & $-0.07 \pm 0.025^{*}$ & 10.4 & $-0.13 \pm 0.032^{*}$ & 10.3 & $-0.13 \pm 0.026^{*}$ & 12.2 \\
\hline & $-1.64 \pm 0.226^{*}$ & 10.4 & $-1.00 \pm 0.300^{*}$ & 10.3 & $-1.07 \pm 0.266^{*}$ & 12.2 \\
\hline \multirow[t]{2}{*}{ Fish } & $0.07 \pm 0.008^{*}$ & -2.58 & $0.06 \pm 0.010^{*}$ & -1.99 & $0.12 \pm 0.010^{*}$ & -3.43 \\
\hline & $0.94 \pm 0.075^{*}$ & -2.58 & $1.08 \pm 0.098^{*}$ & -1.99 & $0.45 \pm 0.098^{*}$ & -3.43 \\
\hline \multirow[t]{2}{*}{ Rice(white) } & $-0.09 \pm 0.024^{*}$ & 14.9 & $-0.04 \pm 0.028$ & 13.8 & $-0.05 \pm 0.023^{*}$ & 17.1 \\
\hline & $-1.19 \pm 0.217^{*}$ & 14.9 & $-1.73 \pm 0.271^{*}$ & 13.8 & $-1.77 \pm 0.237^{*}$ & 17.1 \\
\hline \multirow[t]{2}{*}{ Whole egg fried } & $-0.12 \pm 0.025^{*}$ & 11.2 & $-0.14 \pm 0.030^{*}$ & 10.8 & $-0.17 \pm 0.026^{*}$ & 12.3 \\
\hline & $-1.11 \pm 0.226^{*}$ & 11.2 & $-0.96 \pm 0.290^{*}$ & 10.8 & $-0.65 \pm 0.260^{*}$ & 12.3 \\
\hline \multirow[t]{2}{*}{ Egg without yolk } & $0.07 \pm 0.008^{*}$ & -2.92 & $0.01 \pm 0.009^{*}$ & -1.99 & $0.09 \pm 0.008^{*}$ & -2.49 \\
\hline & $0.11 \pm 0.075$ & -2.62 & $0.73 \pm 0.087^{*}$ & -1.99 & $-0.19 \pm 0.840^{*}$ & -2.49 \\
\hline \multirow[t]{2}{*}{ Raw vegetables } & $0.20 \pm 0.023^{*}$ & -6.16 & $0.05 \pm 0.023^{*}$ & -3.95 & $0.28 \pm 0.024^{*}$ & -7.62 \\
\hline & $1.56 \pm 0.203^{*}$ & -6.16 & $3.15 \pm 0.223^{*}$ & -.3 .95 & $0.81 \pm 0.242^{*}$ & -7.62 \\
\hline \multirow[t]{2}{*}{ Dried fruits } & $0.04 \pm 0.009^{*}$ & -1.85 & $0.07 \pm 0.012^{*}$ & -1.86 & $0.09 \pm 0.010^{*}$ & -2.54 \\
\hline & $0.85 \pm 0.084^{*}$ & -1.85 & $0.41 \pm 0.117^{*}$ & -1.86 & $0.33 \pm 0.105^{*}$ & -2.54 \\
\hline \multirow[t]{2}{*}{ Fresh fruits } & $0.21 \pm 0.022^{*}$ & -6.53 & $0.08 \pm 0.023^{*}$ & -4.64 & $0.26 \pm 0.022^{*}$ & -7.99 \\
\hline & $1.38 \pm 0.191^{*}$ & -6.53 & $2.71 \pm 0.217^{*}$ & -4.64 & $0.91 \pm 0.22^{*}$ & -7.99 \\
\hline \multirow[t]{2}{*}{ Low-fat milk } & $0.11 \pm 0.017^{*}$ & -5.29 & $0.15 \pm 0.021^{*}$ & -5.26 & $0.13 \pm 0.017^{*}$ & -6.19 \\
\hline & $0.81 \pm 0.154^{*}$ & -5.29 & $0.43 \pm 0.200^{*}$ & -5.26 & $0.57 \pm 0.177^{*}$ & -6.19 \\
\hline \multirow[t]{2}{*}{ Cheese } & $0.01 \pm 0.012^{*}$ & -0.15 & $0.08 \pm 0.017^{*}$ & -0.49 & $0.06 \pm 0.014^{*}$ & -1.36 \\
\hline & $1.35 \pm 0.109^{*}$ & -0.15 & $0.27 \pm 0.162$ & -0.49 & $0.64 \pm 0.141^{*}$ & -1.36 \\
\hline \multirow[t]{2}{*}{ Yogurt } & $0.05 \pm 0.014^{*}$ & -3.74 & $0.07 \pm 0.017^{*}$ & -3.46 & $0.09 \pm 0.015^{*}$ & -4.77 \\
\hline & $1.01 \pm 0.125^{*}$ & -3.74 & $0.79 \pm 0.166^{*}$ & -3.46 & $0.62 \pm 0.149^{*}$ & -4.77 \\
\hline \multirow[t]{2}{*}{ Nuts } & $0.09 \pm 0.009^{*}$ & -3.51 & $0.06 \pm 0.010^{*}$ & -2.63 & $0.15 \pm 0.010^{*}$ & -4.70 \\
\hline & $1.16 \pm 0.079^{*}$ & -3.51 & $1.52 \pm 0.098^{*}$ & -2.63 & $0.72 \pm 0.105^{*}$ & -4.70 \\
\hline \multirow[t]{2}{*}{ Gateaux-piments } & $-0.04 \pm 0.011^{*}$ & 5.56 & $-0.04 \pm 0.014^{*}$ & 5.27 & $-0.02 \pm 0.010^{*}$ & 6.94 \\
\hline & $-0.78 \pm 0.099^{*}$ & 5.56 & $-0.69 \pm 0.130^{*}$ & 5.27 & $-1.09 \pm 0.107^{*}$ & 6.94 \\
\hline \multirow[t]{2}{*}{ Light butter } & $0.01 \pm 0.009$ & -0.61 & $0.02 \pm 0.012^{*}$ & -0.46 & $0.04 \pm 0.010^{*}$ & -1.62 \\
\hline & $1.02 \pm 0.081^{*}$ & -0.61 & $0.66 \pm 0.117^{*}$ & -0.46 & $0.59 \pm 0.105^{*}$ & -1.62 \\
\hline \multirow[t]{2}{*}{ Burgers } & $-0.04 \pm 0.018^{*}$ & 7.26 & $-0.07 \pm 0.022^{*}$ & 6.90 & $0.02 \pm 0.016^{*}$ & 9.83 \\
\hline & $-1.31 \pm 0.159^{*}$ & 7.26 & $-1.02 \pm 0.212^{*}$ & 6.90 & $-2.13 \pm 0.161^{*}$ & 9.83 \\
\hline \multirow[t]{2}{*}{ Chips } & $-0.04 \pm 0.017^{*}$ & 7.59 & $-0.08 \pm 0.022^{*}$ & 7.31 & $0.00 \pm 0.015$ & 10.2 \\
\hline & $-1.38 \pm 0.155^{*}$ & 7.59 & $-0.98 \pm 0.209^{*}$ & 7.31 & $-2.13 \pm 0.157^{*}$ & 10.2 \\
\hline \multirow[t]{2}{*}{ Briani } & $-0.10 \pm 0.019^{*}$ & 6.74 & $-0.08 \pm 0.022^{*}$ & 6.12 & $-0.01 \pm 0.016$ & 9.07 \\
\hline & $-0.83 \pm 0.165^{*}$ & 6.74 & $-1.07 \pm 0.208^{*}$ & 6.12 & $-2.10 \pm 0.159^{*}$ & 9.07 \\
\hline \multirow[t]{2}{*}{ Fried noodles/rice } & $-0.08 \pm 0.018^{*}$ & 7.57 & $-0.07 \pm 0.022^{*}$ & 6.95 & $0.01 \pm 0.015$ & 10.1 \\
\hline & $-0.95 \pm 0.163^{*}$ & 7.57 & $-1.14 \pm 0.206^{*}$ & 6.75 & $-2.29 \pm 0.151^{*}$ & 10.1 \\
\hline
\end{tabular}

Note: all regression coefficients were adjusted for participant age and sex

*Significance is indicated with $P$-value $<0.05$, ${ }^{a}$ Regression coefficient, ${ }^{b}$ Standard error

Family Cohesion Score adjusted for family meal frequency (X); Family meal frequency adjusted for family cohesion scores (M)

Meunier et al. [21] showed that the perception of family cohesion by mothers was a predictor of the number of severe hypoglycaemic events through hierarchical regression analyses. Most studies were conducted on family support and the diabetic management of patients and not really family cohesion. Skinner and Hampson [29] have found 
that the general family support was $\mathrm{s}$ significant predictor ( $\beta=0.37, p<0.05$ ) of the efficacy of treatment in controlling diabetes. According to Geffken et al. [11], higher child perceptions of parental warmth and caring were associated with decreased odds of experiencing a diabetic ketoacidosis. In addition, Rosenberg and Shields [27] found that greater maternal perceptions of more secure attachment of adolescents was associated with better glycaemic control.

However, only one study investigated the association between family cohesion and individual dietary intake. Welsh et al. [32] have shown significant negative associations between family cohesion and sweets intake. These findings were somewhat in line with our results that suggested significant negative associations between family cohesion scores and chocolates intake as well as lamb/mutton, white rice, white bread, whole egg fried, gateaux-piments, burgers, chips, briani and fried noodles/rice consumption. Furthermore, our results equally demonstrated significant positive associations between family cohesion and fish, raw vegetables, dried fruits, fresh fruits, low-fat milk, cheese, yogurt, nuts and light butter consumption. Welsh et al. [32] also demonstrated a significant positive association between family meal frequency and family cohesion score which was consistent with our findings as significant positive associations were equally obtained.

Welsh et al. [32] have also hypothesised that family cohesion may be a potential predictor of the association between family meal frequency and individual dietary intake. Although significant associations were demonstrated between family cohesion and dietary intake of sweets in the study, the authors failed to show statistically significant associations between the two variables after adjusting for family meal frequency using hierarchical linear regression models. These findings, therefore, were unlikely to predict any mediation association. Welsh et al. [32] equally obtained a $21 \%$ reduction for the association of family meals and sweets consumption as mediated by family cohesion and the Sobel's test suggested a trend towards a partial mediation. With this interpretation of data, there was no significant evidence for the mediation of family meal frequency and individual dietary intake by family cohesion. In contrast to the results obtained, our results demonstrated strong correlation values between family cohesion score and individual dietary intake after adjusting for family meal frequency and vice versa. A $52.6 \%$ reduction was obtained for the association of family meal frequency and individual dietary intake as mediated by family cohesion score which indicated more or less complete mediation and the findings were confirmed with the Sobel's test. The reason for these differences in findings as compared to the previous study may be due to the smaller sample size used. The different cultural belongings of the
Mauritian population may equally play a role in the research study being investigated.

This study has also taken into account the sociodemographic variables in relation to BMI, skipping meals, glycaemic index, number of meals per day and the average servings of the targeted food items. Our findings indicated that most of the diabetic patients actually consumed three meals per day (52.6 \%) instead of the recommended number of meals, that is, five meals per day $(28.9 \%)$. It was also noted that $60.9 \%$ of the participants did not skip meals and only $39.3 \%$ actually did skip meals. Significant differences were found between age group and skipping meals as well as for education and skipping meals. A decline in breakfast consumption by mostly adolescents has been documented in the United Sates [1]. The reasons for the relationship between skipping meals and age group may be due to the fact that children and adolescents are usually engrossed in irregular activities. Home works, lack of time or any other social gathering may usually contribute to the skipping of meals. Estima et al. [9] have found that breakfast skipping was more common among girls $(12.4 \%)$ rather than boys $(4.5 \%)$. These findings were not in line with our results as no significant differences were noted between gender and skipping meals. It has been reported by Gross et al. [12] that urban students were twice more likely to skip breakfast meals compared to rural and suburban students. In contrast, the present study did not suggest any significant differences between demographic area and skipping meals.

Statistically significant relationships were noted for age group, ethnic group, demographic area, education, occupation and family history in relation to BMI. O'Neil et al. [25] observed a significant trend in age groups for increased consumption of whole grains with lower BMI. Various studies have demonstrated significant relationships between BMI and gender. Lynch et al. [19] have found that BMI z-scores were significantly higher for boys and girls. Srdić et al. [30] and Morimoto et al. [23] have found strong correlation between BMI and percentage body fat among girls comparing to boys. Significant differences between males and females in the BMI categories were equally indicated by James [15]. However these findings were not consistent with the present study as no relationship between gender and BMI was noted.

Interestingly, our results have shown that $97.4 \%$ of the diabetic patients did not have any knowledge about glycaemic index and only $2.6 \%$ were aware of the meaning of glycaemic index. Many studies have shown the importance of consuming a low- glycaemic index meal in order to control blood glucose levels and unfortunately in the present study most of the respondents were unaware of the situation. Yusof et al. [34] has found that a lowglycaemic index diet was associated with significant 
changes in fructosamine level and plasma glucose in Asian patients with Type II diabetes. Jimérez-Cruz et al. [16] have indicated that low-glycaemic index diets have the potential of increasing satiety and decreasing the incidence of diabetes and produce better glycaemic control. Age, occupation, type of diabetes and family history of diabetes were all significantly related to glycaemic index. A proper explanation is necessary to enable the patients to adopt healthy behaviours and to make sensible food choices. Furthermore, adolescents are not exposed to the concept of glycaemic index in schools which means a lower knowledge of the importance of consuming low-glycaemic foods.

Income was a determining variable in the consumption of certain food items. There were statistically significant relationships between income group and consumption of lamb/mutton, brown bread and burgers. Deshmukh-Taskar et al. [7] have found that higher incomes $(>\$ 45,000)$ had lower consumption of burgers or sandwiches. Burnier et al. [6] have found significant associations between high energy intake and income group. The reason for the relationship between lamb/ mutton and brown bread in relation to income in Mauritius may be due to the fact that these food products are relatively expensive and not everybody has the means of affording them. As far as burgers are concerned, they are relatively cheap food items on sale in Mauritius and many individuals are able to readily consume them. Ding et al. [8] have demonstrated that family income was associated with availability of more-healthful food in the home. Furthermore, Mushi-Brunt et al. [24] found no significant differences in fruits and vegetables intake by income status. These findings were much in line with our results which suggested no relationships between income group and fruit and vegetable consumption.

The specificity of the study is the use of a validated method, Family Adaptability and Cohesion Evaluation Scale III (FACES-III) by Baron and Kenny [4], to measure family cohesion which provides a more objective assessment of the present study. Our main findings suggest a strong association between family meal frequency and individual dietary intake of Mauritian diabetic patients and confirm our hypothesis that family cohesion is a plausible mediator of this relationship. The results obtained suggest that not only adolescents but even adults' dietary behaviours are influenced by family characteristics and functioning. Hence, there is a clear demand that both parents and their respective families should benefit from intense behavioural-based interventions that require family members to consider reviewing rules, role modelling, establish limits at mealtimes and adopt positive attitudes when eating together.

Even though our findings precisely demonstrate a relationship between family meal frequency and individual dietary intake, future studies can formulate more effective evaluation tools that accurately measure the frequency of family meals and the quality of meals being served at home. Since not many studies have been conducted on these three parameters, several questions ultimately stem from the results of this study indicating a need for immediate and positive intervention within the field of family meal environment and connectedness.

\section{Conclusions}

A decline in family meals across generations has been observed and this trend may not necessarily be due to the fact that family members do not enjoy eating together. Busy work schedules, children's activities after school, television viewing and new methods of processing and distributing foods such as pre-packaged meals may all be contributing factors to the demise of family members sitting together for a meal. Previous research has investigated and demonstrated the numerous benefits of family meals including greater consumption of healthy foods, adolescent's health status, glycaemic control and lower fasting total cholesterol. Our findings have shown that greater frequency of family meals was significantly associated with greater consumption of healthy foods such as fish, raw vegetables, dried fruits, fresh fruits, low-fat milk, cheese, yogurt, nuts and light butter, that is, better individual dietary intake. Thus it can be recommended that specific nutrition intervention programs encouraging the frequency of more family meals need to be targeted to the diabetic population as well as their respective families. Furthermore, the measurement of family cohesion as a plausible mediator of this relationship adds to the uniqueness of the study. We recorded a $52.6 \%$ reduction for the association of family meal frequency and individual dietary intake as mediated by family cohesion which indicated a more or less complete mediation. Henceforth, it can be concluded that health professionals should take into consideration the connectedness of family members in order to devise innovative nutrition intervention strategies. Therapeutic and psychological interventions, involving family members, can be adopted for the management of diabetes. However, future research in this area is needed to assess other mediators such as parents' rules at home, time spent in food preparation and availability of homemade meals.

\section{Competing interests}

The authors declare that they have no competing interests.

\section{Authors' contributions}

$\mathrm{DR}$ and FM contributed equally in the design of the study. Both authors read and approved the final manuscript.

\section{Acknowledgements}

We extend our deepest gratitude to Dr. Deerajen RAMASAWMY for his statistical guidance and direction throughout the entire project. Our special heartfelt gratitude goes to all the non-governmental organisations and 
clinics throughout the country that have politely agreed to participate voluntarily in the survey.

Received: 14 October 2014 Accepted: 24 June 2015

Published online: 08 August 2015

\section{References}

1. Affenito SG. Breakfast: A missed opportunity. J Am Diet Assoc. 2007;107(4):565-9.

2. American Diabetes Association: Diabetes Statistics, Alexandria. [http:// www.diabetes.org/]

3. Andaya AA, Arredondo EM, Lindsay JE, Eider JP. The association between family meals, TV viewing during meals and fruit, vegetable, soda and chips intake among Latino children. J Nutr Educ Behav. 2009;43(5):113-5.

4. Baron RM, Kenny DA. The moderator-mediator variable distinction in social psychological research: conceptual, strategic and statistical considerations. J Pers Soc Psychol. 1986;51(6):1173-82.

5. Burgess-Champoux TL, Larson N, Neumark-Sztainer D, Hannan PJ, Story M. Unhealthy weight management behaviour among adolescent girls with Type 1 diabetes mellitus: The role of familial eating patterns and weight-related concerns. J Adolesc Health. 2009;35(4):278-89.

6. Burnier D, Dubois L, Girald M. Arguments at mealtime and child energy intake. J Nutr Educ Behav. 2011:43(6):473-81.

7. Combrey T, Neumark-Sztainer D, Story M, Boutelle KN. Parent and family associations with weight-related behaviours and cognitions among overweight adolescents. J Adolesc Health. 2010;47(3):263-9.

8. Deshmukh-Taskar P, Nicklas TA, Yang SJ, Beberson GS. Does food group consumption vary by differences in socioeconomic demographic and lifestyle factors in young adults? The Bogolusa Heart Study. J Am Diet Assoc. 2007;107(2):223-34.

9. Ding D, Sallis JE, Norman GJ, Saelens BE, Harris SK, Kerr J, et al. Community food environment, home food environment, and fruit and vegetable intake of children and adolescents. J Nutr Educ Behav. 2011;1(2):1-5.

10. Estima CCP, Salles Da Costa R, Sichieri R, Pereira RS, Valeira Da Verga G. Meal consumption patterns and anthropometric measurements in adolescents from a low socioeconomic neighbourhood in the metropolitan of Rio de Janeiro, Brazil. Appetite. 2009;52(3):735-9.

11. Fulkerson JA, Kubik MY, Story M, Lytle L, Arcan C. Are there nutritional and other benefits associated with family meals among at-risk youth? J Adolesc Health. 2009;45(4):309-95.

12. Geffken GR, Lehmkuhl M, Walker KM, Storch EA, Heidgerken AD, Lewin A, et al. Family functioning processes and diabetic ketoacidosis in youths with Type 1 diabetes. Rehabil Psychol. 2008;53(2):231-7.

13. Gross SM, Bronner Y, Welch C, Moore-Dewberry N, Paige DM. Breakfast and lunch meal skipping patterns among fourth-grade children from selected public schools in urban, suburban and rural Maryland. J Am Dietetic Assoc. 2004;104(3):420-3.

14. Hing CB, Smith TO, Hooper L, Sone F, Donnell T. A review of how to conduct a surgical survey using a questionnaire. The Knee. 2011;18(4):209-13.

15. International Diabetes Federation (IDF): Diabetes Atlas: The Global Burden [http://www.idf.org/diabetesatlas]. Access 9 May 2015.

16. James DCS. Gender differences in body mass index and weight loss strategies among Americans. J Am Diet Assoc. 2003;103(10):1360-2.

17. Jimerez-Cruz BNM, Loustaunau-Lopez VM, Bacardi-Gascon M. The use of low glycaemic and high satiety index food dishes in Mexico: a low cost approach to prevent and control obesity and diabetes. Nutr Hosp. 2006;21(3):353-6.

18. Larson NI, Nelson MC, Neumark-Sztainer D, Story D, Hannan PJ. Making time for meals: meal structure and associations with dietary intake in young adults. J Nutr Educ Behav. 2009;109(1):72-9.

19. Lau DCW. Diabetes and weight management. Prim Care Diabetes. 2010;1:24-30.

20. Lynch WC, Heil DP, Wager E, Havens MD. Ethnic differences in BMl, weight concerns and eating behaviours: comparison of Native American, white and Hispanic adolescents. Body Image. 2007;48(26):179-90.

21. Mellin AE, Neumark-Sztainer D, Patterson J, Sookalosky J. Unhealthy weight management behaviour among adolescent girls with Type 1 diabetes mellitus: The role of familial eating patterns and weight-related concerns. J Adolesc Health. 2004;35(4):278-89.

22. Meunier J, Dorohy N, Luminet O. Does family cohesiveness and parental alexithymia predict glycaemic control in children and adolescents with diabetes? Diabetes and Metab. 2008;34(5):473-81.
23. Ministry of Health and Quality of Life. The Mauritius Non Communicable Diseases Survey 2009 [http://health.gov.mu/English/Documents/ncd-2009.pdf]. Access 8 June 2015.

24. Morimoto A, Nishimura R, Sano H, Matsudaira T, Miyashita Y, Shirasawa T, et al. Gender difference in the relationship between percent body fat (\% BF) and body mass index (BMI) in Japanese children. Diabetes Res Clin Pract. 2007;78(1):123-5.

25. Mushi-Brunt C, Haire- Joshu D, Elliot M. Food spending behaviours and perceptions are associated with fruit and vegetable intake among parents and their preadolescent children. J Nutr Educ Behav. 2007;39(1):26-30.

26. O'Neil CE, Zanovec M, Cho SS, Nicklas TA. Whole grain and fiber consumption are associated with low body weight measures in US adults: National Health and Nutrition Examination Survey 1999-2004. Nutr Res. 2010;30(12):815-22.

27. Patton SR, Piazza-Waggoner C, Modi AC, Dolan LM, Powers SW. Family functioning at meals relates to adherence in young children with type 1 diabetes. J Paediatr Child Health. 2009;45(12):736-41.

28. Rosenberg T, Shields CG. The role of parent-adolescent attachment in the glycemic control of adolescents with Type 1 diabetes: A Pilot Study. Fam Syst and Health. 2009;27(3):237-48.

29. Salas-Salvado J, Martinez-Gonzalez J, Bullo MA, Ross E. The role of diet in the prevention of type 2 diabetes. Nutr Metab Cardiovasc Dis. 2011;2:32-48.

30. Skinner TC, Hampson SF. Social support and personal models of diabetes in relation to self-care and well-being in adolescents with type 1 diabetes mellitus. J Adolesc. 1998;21(6):703-15.

31. Srdic B, Obradovic B, Dimitric G, Stotic E, Babovic SS. Relationship between body mass index and body fat in children-age and gender differences. Obes Res Clin Pract. 2011;6(2):167-73.

32. Thompson JL, Manore MM, Vaughan LA. The Science of Nutrition. Pearson Benjamin Cummings; 2011.p. 944

33. Welsh EM, French SA, Wall M. Examining the relationship between family meal frequency and individual dietary intake: does family cohesion play a role? J Nutr Educ Behav. 2011;43(4):229-35.

34. World Health Organization. [http://www.who.int/mediacentre/factsheets/ fs312/en - Access 8 June 2015].

35. Yusof BNN, Talib RA, Kamaruddin NA, Karim NA, Chinna K, Gilberson H. A low-Gl diet is associated with a short-term improvement of glycaemic control in Asian patients with type 2 diabetes. Diabetes Obes Metab. 2009;11(4):387-96.

\section{Submit your next manuscript to BioMed Central and take full advantage of:}

- Convenient online submission

- Thorough peer review

- No space constraints or color figure charges

- Immediate publication on acceptance

- Inclusion in PubMed, CAS, Scopus and Google Scholar

- Research which is freely available for redistribution 